Sel f-Organi zed Rot at ing Fi I ament Struct ure in Pl asma in the Large Hel i cal Devi ce After Tracer Encapsul at ed Sol id Pel I et I nj ect i on

\begin{tabular}{|l|l|}
\hline $\begin{array}{l}\text { j our nal or } \\
\text { publ i cat } \mathrm{i} \text { on } \mathrm{t} \text { i } \mathrm{tl} \text { e }\end{array}$ & I EEE Tr ansact i ons on PI asma Sci ence \\
\hline vol une & 42 \\
\hline number & 10 \\
\hline page $\mathrm{r}$ ange & $2512-2513$ \\
\hline year & $2014-10$ \\
\hline URL & ht t p: //hdl . handl e. net /10655/00012927 \\
\hline
\end{tabular}




\title{
Self-organized Rotating Filament Structure in Plasma in the Large Helical Device after Tracer Encapsulated Solid Pellet Injection
}

\author{
Mamoru Shoji, Naoki Tamura, Shigeru Sudo and the LHD Experiment Group
}
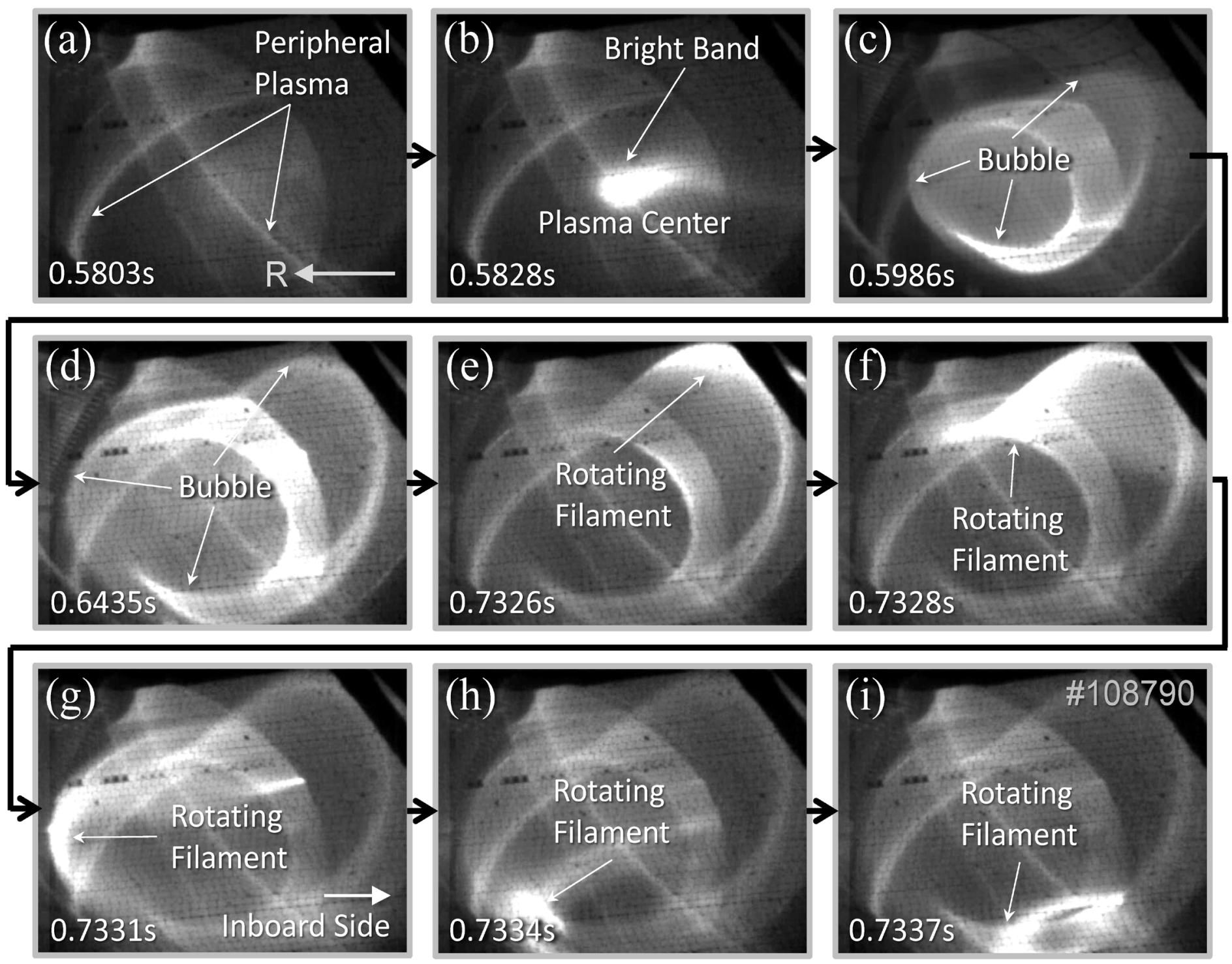

Fig. 1. Sequential images of a tangential view of a LHD plasma after tungsten encapsulated pellet injection, (a) a plasma image before the appearance of the effect of the impurity encapsulated pellet injection, (b) a bright band appeared in the plasma center, (c, d) an expanded uniform bubble-like structure, (e-i) a self-organized bright filament structure rotating on the surface of the bubble-like structure around the plasma center.

Abstract - A small tungsten grain encapsulated in a polystyrene pellet was injected into plasmas in the large helical device. The ionized tungsten was transported and accumulated in the plasma center to drastically drop the central electron temperature. A tangentially viewing fast framing camera observed a bubble-like structure expanding from the plasma center after the pellet injection. After that, a self-organized filament appeared

Manuscript received Manuscript received November 2, 2013; revised April 1, 2014; accepted May 28, 2014.8.

M. Shoji is with the Department of Helical Plasma Research, National Institute for Fusion Science, Toki 509-5292, Japan (e-mail: shoji@nifs.ac.jp). N. Tamura and S. Sudo are with the Department of High Temperature Plasma Physics Research Division, National Institute for Fusion Science, Toki 509-5292, Japan (e-mail: ntamura@lhd.nifs.ac.jp; sudo@ms.nifs.ac.jp).

Digital Object Identifier 10.1109/TPS.2014.2328620 
on the surface of the bubble, and the filament began to rotate around the plasma center, which was stably sustained for $\sim 0.22 \mathrm{~s}$.

\section{Index Terms - Impurity transport, large helical device (LHD), tracer encapsulated solid pellet (TESPEL).}

The Large Helical Device (LHD) is the world's largest super-conducting machine having a heliotron-type magnetic configuration. It has $l / m=2 / 10$ helical coils and three pairs of poloidal coils which magnetically confine plasmas with a major radius of $R=3.50-4.05 \mathrm{~m}$ and an average minor radius of $a=0.5-0.65 \mathrm{~m}$. Magnetic field produced by the superconducting coils forms helically twisted nested magnetic field lines (magnetic surfaces) with an ergodic layer in the plasma periphery. The typical range of the line-averaged electron density and the central electron temperature in the plasmas are in $2-7 \times 10^{19} \mathrm{~m}^{-3}$ and $1.8-3.5 \mathrm{keV}$.

A new diagnostic method with Tracer Encapsulated Solid PELlet (TESPEL) has been developed for impurity transport study by local deposition of the tracer in the plasmas [1], [2]. The TESPEL consists of polystyrene [polymer: $\left(\mathrm{C}_{8} \mathrm{H}_{8}\right)_{n}$ ] as the outer part, and tracers (impurity grains) as the core part. The typical diameter of the outer part of the pellet is $\sim 900 \mu \mathrm{m}$. The most advantageous feature of the TESPEL is production of a localized impurity source as tracers in plasmas. Encapsulated impurity is locally ablated at the position where the normalized minor radius $\rho$ is $\sim 0.8$. The ablated impurity is ionized and transported, which has been observed with various diagnostics such as a soft X-ray pulse height analyzer (SX-PHA), a high-resolution time-resolving soft x-ray multi-channel spectrometer (SOXMOS) and a visible spectrometer array, and so on. The effect of the pellet injection on the fundamental plasma parameter profiles has been monitored with a ten-channel FIR laser interferometer for the electron density measurement, and a multi-point YAG Thomson scattering system for electron temperature measurement. Recently, a tangential viewing visible fastframing camera with a bundled fiber has been installed for monitoring the ablation process of the pellet and the plasma behavior after pellet injection.

Pellets encapsulating a tungsten grain for the tracer with a diameter of $\sim 140 \mu \mathrm{m}$ were injected into a hydrogen plasma, in which the pellet contains $\sim 0.9 \times 10^{17}$ particle number of the tracer with an injection velocity of $\sim 498 \mathrm{~m} / \mathrm{s}$. It caused drastic drop of the electron temperature in the plasma center which is less than the lower limit of the measurements with the Thomson scattering system $(\sim 20 \mathrm{eV})$. It indicates that the presence of a transport mechanism of tungsten ions from the ablation position to the plasma center, enhancing plasma cooling by radiation due to impurity accumulation.
Fig. 1 shows sequential images of the tangential view of a LHD plasma observed with the fast-framing camera after the pellet injection. It was taken in a typical magnetic configuration (the radial position of the magnetic axis $R_{\mathrm{ax}}=$ $3.60 \mathrm{~m}$, the toroidal magnetic field at the magnetic axis $B_{\mathrm{t}}=$ $2.75 \mathrm{~T}$ ). The pellet was injected at $\mathrm{t}=0 \mathrm{~s}$ in this discharge. The left side on the figures corresponds to the outboard side of the torus. The frame rate was set to 20000 frames/s. No significant visible radiation in the plasma center has been observed in normal plasma discharge because of the low impurity content in the plasma. Fig. 1(a) shows visible radiation only in the peripheral plasma before the appearance of the effect of the pellet injection. At $\sim 0.582 \mathrm{~s}$ after the injection, a bright band appeared in the plasma center, as shown in Fig. 1(b). The band expanded from the plasma center, and changed to a uniform bubble-like structure, as shown in Fig. 1(c). It expanded to a magnetic surface around the plasma center, as shown in Fig. 1(d). In this time period, the spectroscopic diagnostics detected transport of tungsten ions into the plasma center, and Thomson scattering system shows that the region with low electron temperature was radially expanded. After a while, a self-organized bright filament structure appeared on the surface of the bubble, as shown in Fig. 1(e). It began to rotate in the poloidal/toroidal directions, as shown in Fig. 1(f)-(i) (it turns counterclockwise on the figure with a rotation frequency of $\sim 760 \mathrm{~Hz}$ ). The images show that the filament is formed along the magnetic field lines on the surface of the bubble. The rotating filament was stably sustained for $\sim 0.22 \mathrm{~s}$. After this, the filament and the bubble structures began to vanish, and it completely disappeared with the rise of the central electron temperature and the drop of the impurity radiation.

In summary, investigation of the rotating filament observed after the tungsten encapsulated pellet is an interesting topic from a viewpoint of research of the formation of a self-organized structure on a magnetic surface in the plasma. It is possible that a helically rotating area with low electron temperature was locally formed along a magnetic field lines around the plasma center, in which accumulated impurities can emit the visible light. Research on the rotating filament can clarify the physical mechanism of the rotation, and may lead to better understanding of physical properties of magnetically confined plasmas.

\section{REFERENCES}

[1] S. Sudo and N. Tamura, "Tracer-encapsulated solid pellet injection system", Rev. Sci. Instrum. 83, 023503 (2012)

[2] S. Sudo, N. Tamura, C. Suzuki, S. Muto, H. Funaba and the LHD Experiment Group, "Multiple-tracer TESPEL injection for studying impurity behavior in a magnetically confined plasma”, Nucl. Fusion 52, 053012 (2012) 